\title{
LA REVOLUCIÓN TECNOLÓGICA Y LOS DESAFÍOS PARA LOS TRABAJADORES
}

THE TECHNOLOGICAL REVOLUTION AND THE CHALLENGES FOR WORKERS

\section{Ángel Estigarribia ${ }^{1}$}

Enviado: 5/2/2021

Aceptado: 20/5/2021

Resumen: En el Paraguay, donde la mano de obra ocupada está mayoritariamente en la agricultura y los servicios, en pocos años pueden desaparecer más de la mitad de los empleos actuales. La irrupción de la revolución tecnológica está produciendo grandes cambios en todos los sectores y en estos dos citados en particular, a escala internacional. La perspectiva de la organización productiva actual es que puede prescindir de la mano de obra de manera acelerada, sobre todo de la poco calificada. La nueva situación emergente implica desafíos para las organizaciones campesinas y sindicatos de trabajadores, para los gobiernos

${ }^{1}$ Facultad de Filosofía, Universidad Nacional de Asunción - Paraguay. Contacto: aestigarribia@yahoo.com 
y sus programas educativos, agrarios y para la planificación estratégica de la economía nacional. Los científicos sociales tienen ante sí la necesidad urgente de estudiar este proceso y de realizar pronósticos, prever consecuencias y orientar con recomendaciones.

Palabras clave: revolución tecnológica; desempleo; tecnología rural, Paraguay.

Abstract: In Paraguay, where the employed labor force is mainly in agriculture and services, in a few years more than half of current jobs can disappear. The irruption of the technological revolution is producing great changes in all sectors and in these two mentioned in particular, on an international scale. The perspective of the current productive organization is that it can dispense with labor in an accelerated manner, especially the low-skilled. The new emerging situation implies challenges for peasant organizations and workers' unions, for governments and their educational and agricultural programs, and for the strategic planning of the national economy. Social scientists are faced with an urgent need to study this process and to make forecasts, foresee consequences and guide with recommendations.

Keywords: technological revolution; unemployment; rural technology; Paraguay. 


\section{Introducción}

"Al ritmo actual de cambio tecnológico, de aquí al final del siglo $X X I$ habremos observado del orden de veinte mil años de progreso

0 , lo que viene a ser aproximadamente lo mismo, unas mil veces más que lo que conseguimos durante el siglo XX».

(Ray Kurzweil)

«Los antagonismos y las contradicciones inseparables del empleo capitalista de la maquinaria no brotan de la maquinaria misma, sino de su empleo capitalista».

(Karl Marx)

Casi el $50 \%$ de los empleos actuales desaparecerían en los próximos años a escala mundial. En América Latina, la cifra pronosticada es aún mayor; el Paraguay, posiblemente, verá extinguirse el $64 \%$ de puestos de trabajo que existen hoy.

El vertiginoso crecimiento y desarrollo de las tecnologías informáticas y robóticas, en las últimas décadas, ha permitido disponer de variados dispositivos, maquinarias y sistemas algorítmicos que abren enormes posibilidades de ahorro de tiempo y esfuerzo humano en la realización del trabajo.

La esperanza de que la calidad de vida puede mejorar, de que el trabajo puede resultar menos tedioso y de que se puede disponer de más tiempo libre, ronda la cabeza de millones de personas, cada vez que aparece el anuncio de un nuevo invento. La oferta de aprendizaje de nuevas profesiones, del manejo de programas informáticos y la posibilidad de trabajar remotamente en cualquier parte del mundo, hacen un nuevo mundo, el de la era de las tecnologías de la información y la 
comunicación (TICs) En poco tiempo, las TICs han introducido a miles de millones de personas a una red de relaciones virtuales personales, comerciales y laborales.

En las revoluciones industriales anteriores, al tiempo que la maquinización reemplazaba el trabajo humano, el desarrollo técnico creaba nuevas fuentes de empleo. El fenómeno actual de la emergencia de la combinación de las TICs con la inteligencia artificial y la robótica -lo que algunos denominan cuarta revolución industrial- parece ser diferente, o por lo menos todavía no hay indicios de que se vayan a generar nuevos y suficientes empleos que sustituyan los que desaparezcan.

En los grandes ciclos de expansión económica, el crecimiento siempre estuvo ligado con el aumento de la demanda de mano de obra, sin embargo, la automatización del trabajo con alta tecnología está cambiando la relación entre la productividad y la utilización de la mano de obra y, con ella, las condiciones laborales. Cada vez se producen más cosas con menos personal. Varios estudios -que se citan más adelante- indican que los empleos, en su concepción actual, cambiarán bastante en los próximos años, y muchos de ellos desaparecerán completamente.

Las diversas dimensiones económicas, comerciales, financieras y laborales de la globalización pudieron ser comprendidas únicamente en referencia al desarrollo de la informática en las últimas décadas del siglo pasado. En la actualidad, los cambios que se están produciendo y los que se esperan en el mundo laboral, deben ser interpretados en el marco de la irrupción de esa poderosa combinación entre TICs, inteligencia artificial y robótica. 
Esta combinación que aumenta significativamente la productividad, al tiempo que elimina empleos, también cambia profundamente el contexto de las relaciones laborales. En América Latina, y en el Paraguay en particular, sectores como la agricultura y los servicios pueden verse muy afectados, en breve tiempo, con su secuela de desempleo, migración, empobrecimiento y crisis social, a falta de desarrollo industrial que pueda absorber la mano de obra desplazada.

En el presente trabajo se intenta mostrar que el fenómeno de la incorporación de alta tecnología y la pérdida de empleos no afecta únicamente a los países altamente industrializados, que la mano de obra barata de los países latinoamericanos no es garantía de asentamientos de nuevas unidades económicas, y que la velocidad de reconversión tecnológica es cada vez más rápida, más barata, más accesible. Sectores de la economía que utilizan mano de obra intensiva como la agricultura y los servicios pueden cambiar completamente en breve tiempo.

La urgencia de analizar el proceso y elaborar estrategias para enfrentar el fenómeno compete a las organizaciones de trabajadores, de productores campesinos, sociedades de emprendedores, técnicos estatales, científicos sociales y por supuesto, al poder político.

\section{Los efectos de la expansión abrupta de la automatización}

Varias décadas atrás, cuando se tenían noticias de un nuevo invento, se daba por hecho que su penetración sería lenta en 
los países latinoamericanos. Los primeros teléfonos móviles eran privativos de millonarios; cuando apareció la pantalla plana de los televisores, el costo inicial era inalcanzable para los trabajadores; el dron parecía más bien juguete para ricos que algo útil económicamente. Rápidamente, y en un proceso casi imperceptible, la mayoría de esos nuevos objetos se volvieron de uso masivo. Aun hoy, cuando se habla de automóviles eléctricos sin conductores humanos, guiados por inteligencia artificial, suena a relato extraterrestre.

Siempre que se lanza al mercado un nuevo invento, incita la curiosidad, impresiona y estimula la imaginación. Una máquina inteligente y autónoma, un dispositivo multiuso, una aplicación que suplanta a varios programas informáticos, normalmente son bienvenidos de manera automática por el público. La reflexión suele llegar después, con su cadena de cuestiones ¿qué consecuencias puede tener en el medio ambiente, en la salud humana, en el mundo laboral?

La llamada globalización del siglo pasado ha sido un fenómeno surgido de la mano de la masificación de la informática, y hubiera sido imposible sin el desarrollo de las tecnologías de la computación e Internet. En el aspecto de la relación laboral, ha producido varias mutaciones como el teletrabajo a escala internacional, el homeworking, las plataformas de intermediación laboral y con ellas una serie de consecuencias que podemos sintetizar en flexibilización o contrarreforma laboral, por un lado, y el problema del desempleo, por el otro. «La globalización, la intensificación de la competencia en todos los mercados de todos los países sirven para legitimar cualquier cosa: la disminución de los salarios reales, el desmantelamiento de las protecciones sociales, la explosión del desempleo, la precariedad de todos 
los empleos, el deterioro de las condiciones de trabajo, etc.» (Gorz, 1998:25-26).

La gran expansión y desarrollo de las tecnologías informáticas y robóticas en los últimos tiempos, ha brindado impactantes novedades, increíbles prestaciones que facilitan la vida, aminoran el trabajo, permiten un abanico de posibilidades de aprendizaje e introducen en un mar de relaciones sociales virtuales a miles de millones de personas. Al mismo tiempo, genera incertidumbres y temor, ya que la automatización -que tiene una enorme capacidad productivadesplaza cada vez más el trabajo vivo y produce desempleo.

Las cuestiones que plantea el informe del BID (Bosch, Pagés, Ripani, 2018:4) son un abanico de incertidumbres. ¿Qué deberían estudiar los jóvenes hoy para el mercado laboral del mañana? ¿Desaparecerán los empleos actuales con la irrupción de los robots? ¿De qué manera transformará la tecnología la forma en que trabajamos? ¿Disminuirá o empeorará el grave problema de la informalidad?

El progreso tecnológico, en las últimas décadas, ha sido tan apabullante, imponente y novedoso que algunos consideran que el mundo se ha adentrado en la cuarta revolución industrial (Bosch, Pagés, Ripani, 2018). Otros investigadores llegan a sostener que la característica actual es distinta de las revoluciones industriales anteriores, que aun eliminando empleos han multiplicado nuevas labores. Hoy está en duda que el boom tecnológico produzca suficientes trabajos como para suplir los que prescinde, y algunos ya advertían de la creciente e irreversible pérdida de empleos ligada a la tecnificación (Rifkin, 1996:27). 
Están cambiando mucho las condiciones laborales, la estructura misma que combina mano de obra y productividad va mucho más allá de la reingeniería de los procesos organizativos. En todos los grandes ciclos de expansión económica han estado combinados el crecimiento con el aumento de la demanda de mano de obra. Tal realidad se ha alterado en los últimos tiempos. Según Goodman (2010), en las expansiones económicas de las décadas de 1950, 1960 y 1970 , los puestos de trabajo aumentaron a una tasa media del 3,5\% anual en el sector privado; pero durante las expansiones económicas de 1980 y 1990, sólo se registró un incremento medio del $2,4 \%$, mientras que en las expansiones de la primera década del siglo XXI, el crecimiento de la ocupación fue en realidad negativo, con un descenso de un $0,9 \%$ anual.

Los datos del país más desarrollado del mundo son ilustrativos. Sherk (2010) encuentra que en la década del 2000 al 2010 los trabajadores industriales en EE.UU. han incrementado la productividad por hora trabajada en un $38 \%$ $y$, mientras que la producción manufacturera se ha mantenido en esa década en niveles estables, el empleo en el sector se ha reducido en más del $32 \%$. Se produce más con menos operarios gracias a la incorporación de nuevas maquinarias y aparatos.

Estudios serios indican que los empleos, tal como los conocemos actualmente, cambiarán bastante en los próximos años e incluso desaparecerán completamente, «el trabajador promedio está perdiendo la carrera contra las máquinas» (Brynjolfsson and McAfee, 2011: pos. 484).

El impacto del desarrollo tecnológico en la pérdida del empleo es indudable. Sucedió en todos los procesos de las 
revoluciones industriales. Los inventos de mecanismos de automatización, efectivamente, desplazaron los trabajos más rudimentarios. La máquina de vapor sustituyó los telares a mano, así como el desarrollo del ferrocarril desplazó el transporte de tracción a sangre, el correo a caballo y finalmente todo el negocio de la cría masiva de equinos. La imprenta puso fin al trabajo de los copistas. Inventos tan simples como el container dejó sin empleo a los estibadores de puertos en todo el mundo.

Donde mejor se ejemplifica el fenómeno de la sustitución del trabajo humano por máquinas es en la agricultura. El tractor y la cosechadora mecánica pusieron fin a millones de tareas agrícolas antiguas. En EE.UU., en 1880, se necesitaban 20 horas/hombre para cosechar 0,4 hectáreas de trigo; en 1916, 12,7 horas/hombre; en 1936, tan sólo 6,1 horas/hombre (Rifkin, 1996:140). La FAO afirmaba, en el 2012 que una máquina mediana moderna podía cosechar entre 7 a 8 hectáreas por día (FAO, 2012). La diferencia con todos esos datos es que hoy, John Deere (2021), el más grande fabricante de máquinas agrícolas y líder en venta en América Latina, ya tiene previsto tractores y cosechadoras autónomos, así como drones pulverizadores e implementos agrarios teledirigidos, lo que significa un cambio cualitativo en las tareas agrícolas por la prescindencia de hasta la mínima expresión de la mano de obra.

Estudios específicos indican que en EE.UU., en 1900, el 40\% de la fuerza laboral trabajaba en la agricultura, pero apenas 2\% en el 2000 (Mc Kinsey Global Institute, 2017:12) y sin embargo, la productividad se ha multiplicado varias veces desde entonces. El empleo industrial ha decrecido, asimismo, y muchos pueden argumentar que, en este caso, la razón es la deslocalización; pero en el empleo agrícola esta 
explicación no sirve, los cultivos no se han relocalizado, más bien se han extendido y el uso de la tierra se ha intensificado.

\section{La combinación entre inteligencia artificial y robótica}

En la actualidad, el acelerado crecimiento de la tecnología de la información, la computación, internet y el salto que ha dado la inteligencia artificial, ha llevado a una combinación eficaz entre lo que se denomina big data y la robótica, que impulsa una simplificación de tareas, una multiplicación en la velocidad de realización y una disminución colosal de su costo. Con toda seguridad, todo esto está produciendo la eliminación de los empleos actuales, pero está en discusión si este cambio significará igualmente la disminución de los empleos globales o si se iran produciendo nuevos y suficientes puestos de trabajo para suplir los que se pierden.

Es cierto que, por ejemplo, la invención de la máquina de vapor y del ferrocarril, supuso la emergencia de millones de nuevos empleos que antes no existían y que superó con creces los trabajos eliminados por los mismos fenómenos. Existen dudas, sin embargo, de que el desarrollo tecnológico actual funcione como las revoluciones industriales anteriores. La absorción del desempleo agrícola fue posible en un país industrializado y exportador como EE.UU, pero el mismo problema, en un país de escaso desarrollo industrial, no tendrá la misma solución. Además, «el cambio tecnológico ha incrementado la demanda relativa de trabajadores de alta calificación, mientras está reduciendo o eliminando la demanda de operarios de baja calificación» (Brynjolfsson \& McAfee, 2011: pos 550), y esto implica un desafío para los programas educativos de los países. Por ejemplo, el trabajo 
agrícola cambiará bastante en las próximas décadas, los operarios tendrán que pasar del uso de la azada y el machete, abruptamente, al manejo de un dron o una serie de cosechadoras desde un computadora portátil.

Si en los dos últimos siglos ha habido un abrumador desarrollo de inventos técnicos y se han producido múltiples cambios tecnológicos y el mundo no ha asistido a la disminución sino a la multiplicación del empleo asalariado, ¿por qué ahora existe la preocupación por el desempleo ligado a la tecnología? Antunes (2009) señala que nunca en la historia ha habido tanta gente empleada y asalariada aunque bajo nuevas condiciones respecto a décadas pasadas.

Según el estudio del BID, ya citado, la revolución industrial a la que estamos asistiendo tiene una diferencia fundamental con las anteriores, y es la velocidad de los cambios (Bosch, Pagés, Ripani, 2018:9). La obsolescencia de la tecnología va al mismo ritmo de los nuevos inventos. La ley de Moore indica que los microcesadores duplican su poder de desempeño aproximadamente cada dos años (Brynjolfsson, and McAfee, 2011: pos 269), pero, por el advenimiento de chips cuánticos (Peper, 2017: 255), quedarán obsoletos en breve tiempo.

El ritmo de los cambios es tan vertiginoso y radical que la transformación de los empleos no se parece -como antañoal paso de la máquina de escribir mecánica a la eléctrica, sino al proceso -ya existente- de dictar un texto a un dispositivo que transcribe sin necesidad de pulsar tecla alguna, y de un país a otro, en tanto que, en medio, toda una serie de trabajos desaparece completamente. Probablemente, los jóvenes que hoy están estudiando, en 5 o 10 años, no encontrarán aquella 
profesión para la cual se estaban preparando (Oppenheimer, 2018).

El denominado estudio de Oxford señala que se perdería el $47 \%$ de los empleos actuales. No sólo los trabajos mecánicos, sino, incluso, aquellos que implican razonamiento y análisis, como los cálculos financieros y el periodismo político (Benedikt y Osborne, 2013:13). Según el BID, en los países de América Latina, la situación sería aún peor, por ejemplo, en República Dominicana (el país con el menor grado de impacto), un $62 \%$ de los empleos estarían en peligro de desaparecer, en el otro extremo, estaría Guatemala con un 75\% de empleos amenazados; Paraguay, perdería el 64\% de los empleos actuales (Bosch, Pagés, Ripani, 2018:13). ¿Podría en verdad ser tan catastrófico o es una exageración?

\section{Dos aspectos claves de la irrupción tecnológica}

Podemos distinguir dos aspectos del desarrollo tecnológico actual y su relación con el empleo. En primer lugar, están las tecnologías de la automatización y, muy ligado a éstas, las de intermediación (Bosch, Pagés, Ripani, 2018).

Entre las tecnologías de automatización se encuentran los robots, las tecnologías de información y comunicación (TICs) y, más recientemente, la inteligencia artificial. Ya es muy conocido lo que implica la robótica en la industria, en los últimos tiempos, se analiza más profundamente el impacto en los servicios y en la agricultura porque, además, es en las últimas décadas que en estos sectores ha dado un salto gigantesco la combinación entre las TICs y la inteligencia artificial. 
Entre las tecnologías de intermediación se hallan las plataformas de transporte compartido (como Uber o Bolt), las de trabajo digital (Upwork, Workana, Freelancer) o las de alquiler de bienes (Airbnb o Turo). Las tecnologías de la intermediación cambian rápidamente el contexto de las relaciones laborales, las plataformas como Uber, Upwork, Bolt, Freelancer, contratan y dan empleo, en todo el mundo, bajo nuevas condiciones: no tienen convenios o contratos colectivos, no se regulan por ninguna legislación laboral ni negocian con sindicato alguno para iniciar operaciones; muy recientemente, en algunos países como el Reino Unido, a partir de la reacción de los trabajadores, han adelantado algunas legislaciones al respecto. (La inédita decisión de Uber en Reino Unido que puede desatar una revolución en la economía gig del mundo. BBC, 17 de marzo de 2021)

El estudio del BID sostiene que otras dimensiones tecnológicas, como la impresión 3D, el blockchain, el internet de las cosas o las innovaciones en biotecnología, aunque tienen asimismo el enorme potencial de cambiar muchas de las condiciones de vida y de trabajo, sus efectos, por lo menos en el mercado de trabajo latinoamericano, no serían aparentemente muy inmediatos (Bosch, Pagés, Ripani, 2018:12).

Pero, a diferencia del análisis anterior, es menester ubicar dentro de las tecnologías de automatización esto que se llama Internet de las cosas (Vongsingthong \& Smanchat, 2014) cuyo potencial es enorme, su desarrollo acelerado y sus consecuencias en el mundo laboral son inconmensurables. $\mathrm{Si}$, hoy, Siri y Alexa ya son compatibles con casi cualquier aparato celular y sirven para poner en funcionamiento dispositivos hogareños, el salto en el mundo productivo está a un paso. Se calcula que todo ser humano está rodeado, al 
menos, por un promedio de 4000 objetos, y que en una década habrá en el mundo aproximadamente 50 mil millones de dispositivos con un sistema de conexión a internet de las cosas (Fernández, 2020). Aparatos como heladeras, acondicionadores de aire, cerraduras, encendido de automóviles, riego del jardín, impresoras, computadoras ya pueden operar solo con la orden de voz o programadas desde el celular. Si se trasladan estas funciones al campo productivo -como ya se está haciendo- la prescindencia de varias funciones que dependían de personas de carne y hueso está a la vuelta de la esquina.

«La aplicación de tecnologías loT [internet de las cosas] que lograrían incrementar en un $70 \%$ la producción global de comida» (Tovar, J. Solórzano Suárez, D., Badillo , A y Rodríguez G, 2019) podría tener efectos inmediatos en el aspecto laboral en los países de fuerte economía rural. Hace posible el manejo remoto de la logística, la detección temprana de vulnerabilidades de los cultivos, la siembra y cosecha en el momento oportuno y de manera automática, con un enorme ahorro de tiempo y esfuerzo de las personas, pues las máquinas pueden operar 24 horas. El problema que presentan estas novedades tecnológicas es que su virtud de ahorrar trabajo humano, en el sistema capitalista se traduce como prescindencia de los trabajadores. No es un problema de la técnica sino del tipo de uso.

\section{Reingeniería de procesos e informatización}

La eliminación de puestos de trabajo no tiene que ver únicamente con tecnología; importa además tener en cuenta los cambios en los procesos organizativos de la actividad 
laboral. Desde mediados del siglo XIX, en poco más de un siglo, desaparecieron más del $90 \%$ de los trabajos agrícolas en EE.UU., y en el siglo XX, entre 1950 y 1980, desaparecieron la mitad de los empleos en la manufactura (Bosch, Pagés, Ripani, 2018:9). De conjunto, el impacto tecnológico y los cambios en las relaciones laborales vía flexibilización laboral, ya sea con las tecnologías de intermediación o vía reingeniería organizativa, ha influenciado enormemente en el mundo laboral y en las expectativas de los trabajadores.

La desigualdad es una de sus consecuencias a corto y mediano plano. Según un estudio de 2016, apenas la mitad de los estadounidenses nacidos en 1980 están mejor que sus padres, comparados con el $90 \%$ de los nacidos en 1940. (Chetty, Grusky, Hell, Hendren, Manduca y Narang, 2016:16)

Las consecuencias en los servicios apenas ha empezado, el desarrollo de sistemas organizativos flexibles y el uso de aplicaciones informáticas y módulos de inteligencia artificial es cada vez más creciente en las empresas de intermediación financiera, casas de cobranzas, comercios al detalle e instituciones bancarias. En los bancos europeos y estadounidenses se prevé el despido de 1.800 .000 empleados en los próximos 10 años (Crowe, 2016).

En muchos países ricos del hemisferio norte, es frecuente culpar a la inmigración por el empobrecimiento y desempleo de sus sectores de trabajadores, o a la relocalización de las empresas en los lugares de menor costo laboral, pero es la combinación entre la reingeniería de procesos y la revolución tecnológica la clave más importante para entender el fenómeno de la pérdida de fuentes de trabajo. Desde la 
popularización en el mundo empresarial del toyotismo, del trabajo just in time y de la reingeniería de procesos ha habido profundos cambios en el mundo laboral (Coriat, 1992).

Estos cambios transformaron mundialmente las relaciones laborales logrando imponer el trabajo a destajo, la tercerización, el pago por productividad en vez del tiempo de trabajo, la flexibilidad de los horarios del trabajador con el sistema justo a tiempo, el fin del pago de horas extras por la compensación de horarios, la transformación del operario especializado en operario de multitareas, el contrato por tiempo determinado (Gorz, 1998).

«Michael Hammer, antiguo profesor del Massachusetts Instituye of Technology (MIT) y primer impulsor de la reestructuración de los procesos empresariales, afirma que la reingeniería produce normalmente como resultado una disminución del $40 \%$ en los empleos de una empresa y del 75 en su masa laboral» (Rifkin, 1996:27).

Lo que produce hoy el proceso acelerado de cambios en el mundo laboral es la combinación de la reestructuración de los procesos de trabajo y la irrupción de la tecnología de la información, la inteligencia artificial y la robótica.

China ha constituido, por varias décadas, una gran factoría de las grandes empresas transnacionales de alta tecnología, en gran medida por la capacidad técnica de los operarios y, en otra, por la baratura del costo laboral. Hoy, es un ejemplo emblemático de que, incluso, la mano de obra de bajo precio es sustituida aceleradamente por la automatización. «La Federacion Internacional de Robótica señala que China está aumentando sus compras de robots industriales $20 \%$ al año, comprando más unidades que los 28 países de la Unión 
Europea juntos».(Oppenheimer, 2018:263) Tan sólo la empresa Foxconn que fabrica en China, entre otros productos, la Iphone de Apple, anunció su plan de incorporar un millón de robots para reemplazar gran parte de su fuerza laboral (Brynjolfsson \& McAfee, 2011: pos 587).

En el marco del ejemplo anterior, los países latinoamericanos no están fuera de la amenaza del salto en el desempleo. $\mathrm{Ni}$ las maquilas, ni los cambios en las legislaciones laborales que precarizan el trabajo serán suficientes para competir con la ola tecnológica. «Ni siquiera en los países más pobres los trabajadores más baratos resultan tan económicos ni tan eficientes como la tecnología inteligente con la que se los sustituye» (Rifkin, 2011:357).

La reacción contra la mecanización, en los inicios de la revolución industrial, no fue amigable, más bien temerosa e incluso hostil. Antes de la prosperidad que produjo, hubo un tiempo de empeoramiento de las condiciones de trabajo y de vida, aumento de la desigualdad, lo que provocó una resistencia de las masas trabajadoras a las máquinas y la tecnología (Frey, 2019). Sin embargo, pasado ese período inicial, la técnica se impuso y significó grandes cambios en el mundo laboral. Si bien es cierto que hizo desaparecer empleos, al mismo tiempo promovió el surgimiento de nuevos trabajos.

La revolución tecnológica actual depara muchos interrogantes y probablemente no va a ser una repetición de las revoluciones anteriores.

Lo cierto es que todavía se conoce muy poco del impacto real de la tecnología sobre el empleo, una vez se tienen en cuenta todos sus alcances. 
Por ahora, los pocos estudios existentes en esa línea, que miden el impacto de la introducción de robots, encuentran efectos negativos en el empleo y/o en los salarios. Sin embargo, la falta de evidencia empírica es notable, en particular, si ponemos el foco en la región [de América Latina y el Caribe] (Bosch, Pagés, Ripani, 2018:15).

\section{El impacto en los países agrarios y de servicios}

En un país como Paraguay, cuya mayor masa de trabajadores empleados trabaja en el sector de servicios (Ministerio de Trabajo, Empleo y Seguridad Social, 2020), que cuenta con muchas empresas dedicadas a diversas operaciones de intermediación, comercio, finanzas, tiene en su derrotero inmediato que muchas tareas en este sector están cada vez más robotizadas o automatizadas: cajeros automáticos, operaciones bancarias on line, ventas vía Internet, aparatos dispensadores de gaseosas, café, golosinas y comida.

Los call center con inteligencia artificial harían superfluas muchas tareas que hoy realizan telefonistas humanos, las entregas domiciliarias realizadas por vehículos autónomos o drones no están tan lejos como se podría pensar. Los trabajadores del transporte serán un sector muy vulnerable en las próximas décadas, por la emergencia de la conducción autónoma. (Brynjolfsson \& McAfee, 2011: pos 721)

La gastronomía, que emplea a buena parte del sector terciario -y que se ha visto extender en iniciativas durante la pandemia- tiene en su futuro inmediato varios retos que 
vienen de la automatización de fábricas de alimentos que comienza a ser una realidad comercialmente viable: robots que hacen pizzas (Food Made Easy, 2020) que fabrican suchis (Issuu, 2020), que producen hamburguesas (The Burn Inn, 2020). No será necesario comprar estos robots, bastará con alquilarlos. Y la gran novedad de las entregas a domicilio, que han tenido un crecimiento sorprendente y están dando empleo a miles de jóvenes, tienen ahora la amenaza de las máquinas robotizadas -que en algunos países dejaron de ser experimentales- como las pizzerías que ofrecen entregas a domicilio con vehículos autónomos (Buss, 2021).

Las empresas de cobranzas, bancos, financieras llevan un largo tiempo automatizándose, y el proceso sigue en curso. La estandarización hace que rápidamente un país latinoamericano tenga las mismas prácticas de un banco norteamericano 0 europeo. Un estudio de EE.UU. mencionado por Oppenheimer (2018:137) - que es perfectamente compatible con Sudamérica- refiere que un cliente bancario promedio interactúa con su banco unas 17 veces por mes, de las cuales 15 consisten en contactos no humanos incluyendo transacciones por celulares, tabletas, consultas telefónicas a call center automatizados y retiros de cajeros automáticos.

Según estudios del Global Perspectives \& Solutions, el 65\% de los costos de una empresa bancaria residen en las sucursales y empleados; por la automatización en curso, se calcula que hasta el 2025 desaparecerán 33\% de las sucursales bancarias en EE.UU. (Digital disruption revisited, 2017:35) y 45\% en América Latina (Oppenheimer, 2018:34). 
Una de las conclusiones del Foro Económico Mundial es la rápida sustitución de empleos humanos por máquinas a escala global y su tendencia la marcan con cifras:

En el 2020 las tareas desempeñadas por seres humanos representaron el $68 \%$ de la actividad productiva a nivel global, mientras que los robots fueron el 32\%. En tres años, sin embargo, la participación de las máquinas aumentará 13 puntos porcentuales al $45 \%$ y los mismos puntos se restarían a los humanos, que terminarían siendo el 55\% de la fuerza laboral (Portafolio, 11 de mayo de 2021).

Para la mayoría seguramente las imágenes inmediatas que evoca la mente cuando se pronuncia el término «tecnología» son celular, tablet, el auto eléctrico autónomo que circula por la ciudad, la fábrica con robots o bancos y supermercados que funcionan con dispositivos de inteligencia artificial, sin cajeros humanos. Sin embargo, es probable que el mayor impacto social se produzca en el campo, en las faenas agrícolas y ganaderas.

Algunos estudios indican que varios aspectos del desarrollo tecnológico tendrán uso inmediato, primeramente en la agricultura y en el transporte por carretera. Es relativamente más fácil y menos riesgoso el funcionamiento de vehículos autónomos en las zonas rurales, así como el accionar de tractores y maquinarias agrícolas automáticas. Por ahora los camiones autónomos están en fase experimental en algunas rutas, pero los analistas de tecnología calculan que en el 2025, el $33 \%$ de los camiones que transitan por las rutas serán autónomos (Farhard, 2016) Aunque puede resultar muy optimista, el proceso va en esa dirección. De hecho, los 
camiones autónomos fuera de carreteras ya se usan en países sudamericanos que tienen producción minera a cielo abierto como Chile (Magri, 2014). «Actualmente estamos en un contexto de transporte autónomo. El siguiente paso es llegar a la mina autónoma, que es aquella donde no hay personas en las operaciones» (Komatsu aumentará su flota de camiones autónomos en 2020. 12 de Septiembre de 2019). Si un camión autónomo puede operar en una mina a cielo abierto, puede hacer lo mismo en zonas remotas de producción forestal, en el transporte de soja y trigo desde la línea de cosecha hasta los silos, es decir, por ahora fuera de las rutas de mucho tránsito.

La pérdida de empleo en el sector rural ha sido la constante histórica mundial desde la introducción de tecnología. «En el siglo XIX, alrededor del $25 \%$ de toda la labor en la agricultura consistía en el trillado de granos. Esta tarea fue automatizada en 1860» (Brynjolfsson \& McAfee, 2011: pos 570). Las consecuencias de la automatización actual no serán diferentes, sus implicaciones conllevan mayor concentración de la tenencia de la tierra, más migración a la ciudad.

Puede que algunos sostengan que, por la pobreza y el atraso, por un buen tiempo, la incorporación de nuevas maquinarias y de nuevos dispositivos técnicos no sea tan rápida. Sin embargo, el abaratamiento tendencial nos puede indicar lo contrario. ¿Cuánto tiempo tardó para que el celular sea de uso masivo y en todas las clases sociales? ¿Cuántos años llevó para que las motocicletas inundaran el pobre sector rural del país? Además, el conocimiento, las novedades, hoy son de fácil apropiación y las posibilidades de aprendizaje del uso de nuevos dispositivos son inmensos. La NASA y el presidente de EE.UU. tenían menos acceso a la información 
hace dos décadas atrás que hoy cualquiera con un celular (Oppenheimer, 2017:26).

El gremio de los horticultores -que hoy lucha cada vez que cae el precio de sus productos- no podrá dejar de ver en las redes sociales y en YouTube la cada vez mayor variedad de implementos automatizados. En el sector hortícola, que utiliza mucha mano de obra, la incorporación de alta tecnología seguramente está muy próxima, quizá sea muy costosa una cosechadora de tomates (Mastermac, 15 de Mayo de 2021) que puede trabajar centenares de hectáreas de cultivos por día, pero están otras como las máquinas low cost que pueden operar incluso en los invernaderos de polipropileno (Le, Ponnambalam, Gjevestad \& From, 2020). En el mercado latinoamericano ya están disponibles cosechadoras de tomates, de yerba mate (Quaizel, 2020), y en prototipo la de cítricos (La Universidad patenta una pequeña cosechadora de cítricos, 18 de junio de 2020) que se suman a los ya muy conocidas de cereales y oleaginosas.

\section{Plataformas informáticas y flexibilización laboral}

La automatización robotizada elimina empleos, y las tecnologías de la intermediación cambian profundamente el contexto de las relaciones laborales, pero también despiertan de la latencia sectores antes no productivos. «En definitiva, un mayor uso de capital y trabajo que antes estaban ociosos ahora puede tener efectos positivos en el crecimiento de los países. » (Bosch, Pagés, Ripani, 2018:16). Las plataformas convirtieron miles de autos particulares en servicios de transporte del tipo Bolt, Uber y Muv. Jóvenes con motocicletas encuentran empleo en los servicios de entregas a domicilio o 
deliverys dependientes de plataformas como Pedidos Ya, Monchis, sobre todo con el advenimiento de la pandemia. El fenómeno de los call-center con sus centenares de empleados tiene que ver con la multiplicación de los sistemas de ventas en línea.

Servicios como Airbnb facilitan el alquiler de viviendas o habitaciones sin uso por parte de las familias y pueden constituir fuentes de ingresos en las zonas rurales del país en el marco del turismo interno. Y así como estas tecnologías de intermediación tienen un efecto importante, para la creación de nuevos empleos o fuentes de ingresos, sobre todo a tiempo parcial, al mismo tiempo imponen condiciones de trabajo precarios como las que establecen aquellas plataformas del tipo Up work, Freelancer y otros que intermedian empleos en todo el mundo.

La flexibilidad en el uso del tiempo es una de las características de estas empresas intermediarias que operan en plataformas a través de Internet. Además y sobre todo, la reducción de los costos sociales para las empresas es una de las novedades importantes ya que no reconocen una relación laboral y, por lo tanto, pagan a destajo, no hay salario ni prestaciones sociales.

Muchos servicios, puestos a través de plataformas, actúan de manera parecida a las empresas relocalizadas: bajo costo salarial, pero sin necesidad de trasladar las empresas o dependencias a otro país. Los trabajadores no migran, pueden trabajar desde sus casas para empresas del primer mundo que no tienen que lidiar con el problema de contratar en negro en su propio país, pero pueden pagar como si fuera. 
La nueva incorporación a la estrategia de control del trabajo a través de plataformas montadas en Internet es lo que ha venido a llamarse el modelo Uber (uberización) de explotación laboral.

Por representar un símbolo de un nuevo modelo económico, el nombre la firma Uber se prestó para crear esta expresión. Esta expresión se usa para definir los nuevos modelos de negocios en los cuales particulares pueden efectuar transacciones económicas vía plataformas accesibles desde aplicaciones que se encuentran en sus celulares inteligentes o en sus computadores. Además de Uber, los últimos años han experimentado una explosión de oferta de plataformas que van desde el alojamiento con AirBnB hasta plataformas de intermediación financiera, otras dedicadas a los servicios domésticos, a los servicios jurídicos, etc. (Bardey, 2015).

La clase media propietaria de pequeñas tiendas comerciales ha recibido varios impactos negativos como producto del desarrollo tecnológico. En los últimos tiempos, los servicios a través de plataformas han eliminado varias fuentes de trabajo. Netflix eliminó completamente los locales de alquileres de DVD; Spotify e Itunes, hicieron desaparecer los negocios de ventas de CD, incluido los que hacían copias ilegales, más baratas. Los servicios de celulares, pusieron fin a los locutorios y los cyber-cafés que ofrecían servicios de acceso a Internet. Del mismo modo, los negocios que vendían programas informáticos en CD y DVD van desapareciendo porque los software y juegos hoy se venden o se usan en línea. 
Hay mucha discusión respecto al futuro del trabajo. Nadie duda de que la pérdida de empleo suscitado por el desarrollo tecnológico sea real; sí hay dudas de su profundidad y gravedad. Lo que es claro, por lo menos en las experiencias de los países desarrollados, es la pérdida de fuentes de trabajo en la agricultura, debido a la incorporación de maquinarias, que no generan empleos sustitutos en el campo. En los países industrializados los millones de empleos perdidos en las faenas del campo encontraron sustitutos en la manufactura y los servicios en los siglos, XIX y XX. (Brynjolfsson \& McAfee, 2011: pos 726).

En las últimas décadas, aun en los sectores de industria y servicios, los cuales han sido mejores creadores de empleo, la tendencia a la supresión del trabajo vivo y la sustitución por tecnología es creciente.

El siguiente paralelismo es válido: si en el período de la empresa taylorista y el fordismo la fuerza de las empresas era medida según el número de trabajadores y trabajadoras que actuaban en las mismas; en la era actual de las empresas flexibles y digitalizadas del mundo financiarizado, estas son consideradas más productivas, con mayor proyección global y con perfiles más transnacionales en la medida en que cuenten con menos trabajo vivo (Antúnez, 2020, p. 15).

\section{Las perspectivas del empleo rural}

En los países industrializados, si bien se han perdido muchos empleos en el sector rural, el carácter de su desarrollo, ligado 
al sector secundario, ha permitido balancear estas pérdidas con la creación de fuentes de trabajo en las ciudades fabriles. No es lo mismo en los países de producción primaria dominante.

En el caso de los países menos industrializados y con un gran segmento de la población involucrada en trabajos en el sector rural, sobre todo en la agricultura y ganadería, la incorporación de tecnología moderna: tractores, cosechadoras, fumigadoras autónomas, sistemas de riego y fertilización controlados por computadoras implicará una fuerte tenencia al desempleo pues es un sector acostumbrado al uso intenso de la mano de obra.

Paraguay, que tiene una gran parte de su población todavía empleada en la agricultura, enfrenta varios desafíos con respecto a los cambios tecnológicos: reformar la política educativa, diseñar y desarrollar nuevas formas de empleo y prever una nueva ola migratoria del campo a la ciudad con todas las consecuencias que esto supone. Según el informe de gestión del Ministerio de Trabajo, Empleo y Seguridad Social (2020) la agricultura y los servicios son los que más emplean trabajadores. En el sector primario, conformado por agricultura, ganadería, caza y pesca, trabajan 780.792 personas. En el sector secundario, que incluye la industria manufacturera y la construcción: 598.416; en el terciario o de servicios: 1.938 .998 trabajadores.

Por ahora, las tecnologías de punta están ligadas a los grandes cultivos empresariales. Pero, hay que tener en cuenta que así, como las computadoras, que al principio parecían que podrían ser utilizadas únicamente por las grandes empresas industriales y de servicios, por su costo y complejidad, masificarán rápidamente su uso. Tampoco se 
puede pensar que la única vía de acceso es que cada productor deba comprarse sus maquinarias. Ya es tendencia en varios países que estas puedan ser alquiladas, y los pequeños y medianos productores puedan acceder a ellas sin necesidad de desembolsar mucho dinero. El fenómeno del pool agrario en la Argentina es un ejemplo cercano

En sistemas agrarios de producción empresarial que se concentraban en la rotación del capital y su desplazamiento de fijo a variable, la búsqueda de beneficios rápidos, el uso de la tecnología para reemplazar mano de obra y, en este caso específico, el abandono de la idea de apropiación permanente de la tierra y su reemplazo por el alquiler temporario. Como resultado de estos cambios, creció enormemente la terciarización de los servicios productivos, como la siembra, la fumigación y la cosecha, y apareció con fuerza el llamado contratista, una empresa que posee maquinarias de todo tipo y que recorre los campos ofreciendo sus servicios (Reboratti, 2010:68)

El desarrollo desigual crea brechas, pero la misma desigualdad hace posible combinaciones complejas, dada la velocidad de los cambios en el mundo de hoy. La modernización puede, de esta manera, dar un salto, con sectores campesinos que podrían pasar a manejar maquinarias hibridas y a control remoto sin haber pasado por el uso del tractor. La demanda de destrezas tecnológicas pondrá a la orden del día la necesidad de capacitación en tecnología en el mundo agropecuario, lo que seguramente impulsará una revisión total de los programas de estudios escolares en todos los niveles. La emergencia de nuevos tipos de organizaciones productivas seguramente provocará 
crisis en las sociedades rurales tradicionales y el desplazamiento de pequeños productores que no puedan adecuarse a los cambios aumentará la brecha de desigualdad y profundizará gravemente la migración a los cinturones marginales de las ciudades.

\section{Conclusión}

Los empleos que se pierden en el sector agrícola en virtud del impacto tecnológico no se recuperan en el mismo sector: es la constatación de una tendencia internacional. Las destrezas necesarias para el nuevo escenario que se acerca aceleradamente serán muy distintas de las que poseen actualmente el trabajador rural.

La tecnificación posiblemente aumentará la concentración de la tierra en detrimento del pequeño productor. Los pool agropecuarios tendrán un fuerte crecimiento. Los empresarios del agronegocio, sin tierra, sin maquinarias, sin implementos pero con capital para alquilar todo lo necesario para producir con bajo costo de mano de obra, son los ejemplos más cercanos -Brasil y Argentina- del tipo de producción agrícola no solo de commodities para exportación sino también de la producción de alimentos para el mercado interno.

Al no avizorarse una política de industrialización en el corto y mediano plazo en el Paraguay, la migración del campo a la ciudad va a ser muy caótica, engrosará el cinturón de pobreza de las ciudades. Los emprendedores en el sector terciario se verán sometidos a una fuerte competencia de aquellas empresas que incorporan tecnología y prescinden de mano 
de obra, en los rubros financiero, comercial, gastronómico y de servicios de entregas a domicilio.

Las plataformas de intermediación laboral internacional permitirán el acceso a una fuente de empleo a un buen sector de profesionales de diseño gráfico, arquitectura, ingeniería, edición y de otras labores posibles de realizar a distancia. Asimismo, crecerán en su cobertura las plataformas de alquileres de autos y de servicios de entregas en detrimento de las pequeñas empresas que incursionaron en el rubro. De esta forma aumentará el número de trabajadores a tiempo parcial, sin cobertura del seguro social y sin contrato como empleados dependientes.

Ardua labor espera a los responsables de las políticas económicas y laborales, a las organizaciones de trabajadores, a las organizaciones campesinas, y a los estudiosos de las ciencias sociales para responder a esta nueva realidad.

Desde ya, implica desafíos para las organizaciones campesinas y sindicatos de trabajadores, en el plano organizativo y legislativo. Desafío para el gobierno y sus programas educativos, agrarios y sobre todo para la planificación estratégica de la economía.

Los científicos sociales se enfrentan asimismo a la necesidad de realizar pronósticos, prever consecuencias y orientar con líneas políticas de empleo, reforma agraria, financiación productiva, programas urbanísticos de mitigación de la migración interna, legislación para los nuevos fenómenos del mundo laboral. 
El proceso de cambios ya está en curso, y el tiempo de espera para la adecuación es cada vez más corto, con lo que aumenta la urgencia de analizar y afrontar este fenómeno.

\section{Referencias}

- Antúnes, R. 2020. ¿Cuál es el futuro del trabajo en la era digital? Revista Observatorio Latinoamericano y Caribeño Vol. 4, No 1, pp, 13-22

https://publicaciones.sociales.uba.ar/index.php/observatori olatinoamericano/article/view/5465/4849

2009 Diez tesis sobre el trabajo del presente (y el futuro del trabajo) en Trabajo, empleo, calificaciones profesionales, relaciones de trabajo e identidades laborales. Vol. I pp. 29-43. Buenos Aires. CAICyT CLACSO.

http://biblioteca.clacso.edu.ar/clacso/gt/20160216040822/0 4.pdf

- Bardey, D. 2015. ¿Qué significa la "Uberización” de la economía? ¿Cuáles son sus consecuencias para Colombia? Foco Económico.

http://focoeconomico.org/2015/08/04/que-significa-lauberizacion-de-la-economia-cuales-son-susconsecuencias-para-colombia-por-david-bardet/

- Brynjolfsson, E and McAfee, A. 2011. Race Against the Machine. How the Digital Revolution is Accelerating Innovation, Driving Productivity, and Irreversibly Transforming Employment and the Economy. Lexington, Massachusetts, Digital Frontier Press. Ebook created by www.ebookconversion.com. https://www.amazon.com//es/Erik-Brynjolfsson-ebook/dp/B005WTR4ZI 
- Benedikt, C. Osborne, M. 2013. The future of employmente: how susceptible are Jobs to computerisation. September 17, 2013.

https://www.oxfordmartin.ox.ac.uk/downloads/academic/Th e_Future_of_Employment.pdf

- Bosch, M.; Pagés, C.; Ripani, L. 2018. «El futuro del trabajo en América Latina y el Caribe: ¿Una gran oportunidad para la región?». Banco Interamericano de Desarrollo.

https://publications.iadb.org/publications/spanish/document /El_futuro_del_trabajo_en_Am\%C3\%A9rica_Latina_y_el_ Caribe_Una_gran_oportunidad_para_la_regi\%C3\%B3n_v ersi\%C3\%B3n_para_imprimir.pdf

- Buss, D. 2021. Domino's AV Test With Nuro Is A Picture Of Future Pizza Delivery. Forbes. https://www.forbes.com/sites/dalebuss/2021/04/29/domino s-av-test-with-nuro-is-a-picture-of-future-pizzadelivery/?sh=1493657f5290

- Chetty, R.; Grusky, D.; Hell M.; Hendren, N.; Manduca, R. and Narang, J. 2016. The Fading American Dream: Trends in Absolute Income Mobility Since 1940. https://inequality.stanford.edu/sites/default/files/fadingamerican-dream.pdf

- Coriat, B. 1992. Pensar al revés. Trabajo y organización en la empresa japonesa. Madrid, Siglo XXI.

- Crowe, P. 2016. CITI: The 'Uber moment' for banks is coming - and more than a million people could lose their Jobs. Businees Insider https://finance.yahoo.com/news/citi-uber-moment-bankscoming-214925606.html

- F.A.O. 2012. Cosecha de granos. Depósito de documentos de la $\mathrm{FAO}$, disponible en Internet en http://www.fao.org/docrep/x5051s/x5051s02.htm 
- Farhard, M. 2016. Fort he debaters: what shall we do about the tech careening our way, New York Times 22 de setiembre de 2016.

https://www.nytimes.com/2016/09/22/technology/for-thedebaters-what-shall-we-do-about-the-tech-careening-ourway.html)

- Fernandez, R. 2020. Dispositivos conectados a Internet en el mundo 2018-2030 https://es.statista.com/estadisticas/517654/prevision-de-laevolucion-de-los-dispositivos-conectados-para-el-internetde-las-cosas-en-elmundo/\#: :text=De\%20cumplirse\%20las\%20previsiones\% 2C\%20el,millones\%20en\%20el\%20a\%C3\%B10\%202030.

- Food Made Easy. 2020. https://www.hellopicnic.com/

- Frey, C. 2019. The Technology Trap: Capital, Labor, and Power in the Age of Automation. Princeton University Press. Kindle Edition.

- Goodman, P. 2010. Despite signs of recovery, chronic joblessness rise. New York Times,.

https://www.nytimes.com/2010/02/21/business/economy/2 1unemployed.html

- Gorz, A. 1998. Miserias del presente, riqueza de lo posible. Buenos Aires, Paidós.

- Issuu. 2020. https://issuu.com/sushirobots.eu/docs/catalogue-es-sp

- Deere, J. 2021. El futuro de la agricultura. https://www.deere.es/es/agricultura/el-futuro-de-laagricultura/

- Le, T. Ponnambalam, V. Gjevestad, J. From, P. 2020. A low-cost and efficient autonomous row-following robot for food production in polytunnels. Journal of Field Robotics. March 2020 pp-309-321. https://doi.org/10.1002/rob.21878. 
- Mc Kinsey Global Institute. 2017. A future thk Works: automation, employment, and productivity. https://www.mckinsey.com/ /media/mckinsey/featured\%20 insights/Digital\%20Disruption/Harnessing\%20automation\% 20 for\%20a\%20future\%20that\%20works/MGI-A-future-thatworks-Full-report.ashx

- Magri, J. 2014. Efectos de la incorporación de tecnologías autónomas en el diseño y la planificación minera. Universidad de Chile. Facultad de Ciencias Físicad y Matemáticas. Departamento de Ingeniería de Minas. Santiago de Chile. http://repositorio.uchile.cl/bitstream/handle/2250/131395/Ef ectos-de-la-incorporacion-de-tecnologiasautonomas....pdf?sequence $=1$ \&isAllowed $=y$ )

- Ministerio de Trabajo, Empleo y Seguridad Social (2020) Informe de gestión año 2020. https://www.mtess.gov.py/application/files/1716/0926/6636 /informe_gestion_2020.pdf

- Oppenheimer, A. 2017. ¡Crear o morir! La esperanza de América Latina y las cinco claves de la innovación. México, Debate.

- Oppenheimer, A. 2018. ¡Sálvese quien pueda! El futuro del trabajo en la era de la automatización. Buenos Aires, Debate.

- Peper, F. 2017. The End of Moore's Law: Opportunities for Natural Computing? New Genereration Computing. $N^{\circ} 35$, 253-269 (2017). https://doi.org/10.1007/s00354-017-00204

- Portafolio. 2021. Máquinas desplazarán en 2025 a 85 millones de puestos de trabajo. https://www.portafolio.co/economia/empleo/maquinasdesplazaran-en-2025-a-85-millones-de-puestos-detrabajo- 
551861?utm_source=exacttarget\&utm_medium=enlace\&ut $\mathrm{m} \_$campaign $=$Boletines_IniciandoEIDia

- Quaizel, G. 2020.. Las cosechadoras de yerba mate ganan presencia. Noticias agropecuarias, Posadas. https://www.noticiasagropecuarias.com/2020/09/22/lascosechadoras-de-yerba-mate-ganan-presencia/

- Reboratti, C. 2010. Un mar de soja: la nueva agricultura en Argentina y sus consecuencias. Revista de geografía Norte Grande. №.45 pp. 63-76. Santiago, mayo 2010. https://scielo.conicyt.cl/scielo.php?script=sci_arttext\&pid=S 0718-34022010000100005

- Rifkin, J. 1996. El fin del trabajo. Nuevas tecnologías contra puestos de trabajo: nacimiento de una nueva era. Buenos Aires, Paidós.

- 2011. La tercera revolución industrial. Cómo el poder lateral está transformando la energía, la economía y el mundo. Barcelona, Paidós.

- Sherk, J. 2010. Technology Explains Drop in Manufacturing Jobs. https://www.heritage.org/jobs-andlabor/report/technology-explains-drop-manufacturing-jobs

- The Burn Inn. 2020.

https://www.theburnin.com/startups/miso-robotics-burgerflipping-robot-only-costs-3-per-hour-2020-2/

- Tovar, J.; Solórzano Suárez, D.; Badillo Rodríguez, A,; y Rodríguez, G. 2019. Internet de las cosas aplicado a la agricultura: estado actual, Lámpsakos, N²2, pp. 86-105 (julio-diciembre, 2019). https://doi.org/10.21501/21454086.3253

- Vongsingthong, S y Smanchat, S. 2014. Internet of things: a review of applications \& technologies. Suranaree Journal of Science and Technology. Vol. 21. № 4, pp. 359374 . Suaranaree University of Technology https://doi.nrct.go.th//ListDoi/listDetail?Resolve_DOI=10.14 456/sjst.2014.38 